\title{
Non ischaemic causes of lacunar syndromes: prevalence and clinical findings
}

\author{
NICOLETTA ANZALONE,* GIANLUCA LANDI \\ From the Neurological Clinic, Ospedale Policlinico, Milano, Italy
}

SUMMARY To evaluate the prevalence of lacunar syndromes due to non ischaemic causes 97 consecutive patients with recent ( $<72$ hours) onset of a recognised lacunar syndrome were studied. Investigations showed that nine cases were due to non ischaemic pathologies (four primary intracerebral haemorrhages, one rupture of a mycotic aneurysm, one cerebral abscess, one subdural haematoma, one glioblastoma, one multiple sclerosis). Clinical features did not allow a separation of non ischaemic from ischaemic patients; however, hypertension was significantly more frequent in the latter group, and its positive predictive value in identifying ischaemic patients was $96.6 \%$. Lacunar syndromes due to non ischaemic causes are not rare; since CT scan allowed appropriate treatment in two patients and demonstrated contraindications to antithrombotic therapy in five others, its early use appears warranted in patients with acute lacunar syndromes, particularly if normotensive.

According to Fisher's pathological studies, ${ }^{2}$ lacunes are small deep cerebral infarcts which result from occlusion of intracranial penetrating arterioles, caused by hypertension. This author also described several clinical syndromes ${ }^{3-7}$ which he considered distinctive for these infarcts, and which therefore have been related to the same pathogenesis. Later work has shown that lacunar syndromes may be caused also by embolism from the heart ${ }^{8}$ or carotid artery occlusion. Since these patients often present with reversible neurological deficits, they are candidates for long-term treatment with antithrombotic drugs. However, several papers have reported the occurrence of lacunar syndromes due to non ischaemic causes. ${ }^{10-12}$ Yet, both prevalence and clinical characteristics of these cases have never been systematically investigated. We therefore evaluated a series of patients presenting with recent acute onset of a typical lacunar syndrome.

\section{Patients and methods}

We prospectively included a consecutive series of 97 patients

*Present address: Department of Neuroradiology, Ospedale San Raffaele, Milano, Italy.

Address for reprint requests: Dr Gianluca Landi, Clinica Neurologica, Ospedale Policlinico, Via F. Sforza 35, 20122 Milano, Italy.

Received 23 December 1988 and in revised form 29 March 1989. Accepted 6 April 1989 visited by one of us at the emergency room of our hospita夜 from 1 August 1983 until 31 January 1987, who presente with a recognised lacunar syndrome (pure moto® hemiparesis, pure sensory stroke, ataxic hemiparesis dysarthria-clumsy hand syndrome, hemiballism) of recent ( $<72$ hours) onset and no history of previous stroke.

All patients received neurological and general examina tions, and they were screened for the presence of cerebros vascular risk factors such as hypertension (blood pressure higher than $160 / 90 \mathrm{~mm} \mathrm{Hg}$ or regular use of antihypertensive drugs), smoking (10 or more cigarettes per day), diabetes (fasting blood sugar levels higher than $140 \mathrm{mg} \%$ or regular use of antidiabetic drugs), hyperlipidaemia (plasma cholesterol and/or triglycerides higher than $280 \mathrm{mg} \%$ and $200 \mathrm{mg} \%$, respectively), cardiac disease (valvular and coronary heart disease, atrial fibrillation, or other clinically relevant arrhythmias) and occurrence of previous transient ischaemic attacks (TIA).

A CT scan was performed in all patients on admission using an EMI 1010 unit, and it was repeated later if appropriate. All patients were then followed up until death or for at least three months. Patients whose CT scan showed either no focal abnormality or appropriate small deep hypodense areas and who experienced no further symptoms were diagnosed as having lacunar infarction. Patients with a pathological CT scan which was not compatible with lacunar infarction and those who presented a worsening of their symptoms or new attacks underwent further studies as deemed appropriate (CT scan with intravenous contrast in eight cases, CSF examination in one case, cerebral angiography in two cases, cortical evoked potentials in one case, and MRI of the head in one case), until a final diagnosis was established. 
Table 1 Summary of clinical features

\begin{tabular}{lcl}
\hline & Ischaemic lacunar syndromes $(\boldsymbol{n}=88)$ & Non ischaemic lacunar syndromes $(\boldsymbol{n}=9)$ \\
\hline Mean age (range) (yr) & $65 \cdot 3(20-87)$ & $62 \cdot 8(25-85)$ \\
Sex & $55 \mathrm{M} / 33 \mathrm{~F}$ & $7 \mathrm{M} / 2 \mathrm{~F}$ \\
Onset: sudden & $52(59 \cdot 1 \%)$ & $8(88 \cdot 9 \%)$ \\
gradual, stepwise or fluctuating & $36(40 \cdot 9 \%)$ & $1(11.1 \%)$ \\
Headache (preceding or at onset) & $7(8 \cdot 0 \%)$ & $1(11 \cdot 1 \%)$ \\
Type of lacunar syndrome: & $67(76 \cdot 1 \%)$ & $7(77 \cdot 8 \%)$ \\
$\quad$ Pure motor hemiparesis & $6(6 \cdot 8 \%)$ & 0 \\
Pure sensory stroke & $8(9 \cdot 1 \%)$ & $2(22 \cdot 2 \%)$ \\
Ataxic hemiparesis & $5(5 \cdot 7 \%)$ & 0 \\
Dysarthria-clumsy hand & $2(2 \cdot 3 \%)$ & 0 \\
Hemiballism & Lacunar infarct: 88 & Primary intracerebral haemorrhage: 4 \\
Final diagnosis & & Ruptured mycotic aneurysm: 1 \\
& & Multiple sclerosis: 1 \\
& & Cerebral abscess: 1 \\
& & Subdural haematoma: 1 \\
\hline
\end{tabular}

\section{Results}

Out of our 97 patients, 74 presented with a pure motor hemiparesis, six with pure sensory stroke, 10 with ataxic hemiparesis, five with dysarthria-clumsy hand syndrome, and two with hemiballism. In nine cases $(9 \cdot 3 \%)$ investigations revealed a non ischaemic pathogenesis of the symptoms. A non enhanced CT scan demonstrated four small haemorrhages in the basal ganglia: two of these patients were hypertensive, whereas extensive investigations revealed no apparent cause in the other two cases. Another non-hypertensive haemorrhage was located deep in the right parietal lobe; in this case, the presence of an aneurysm at angiography together with echocardiographic evidence of mitral valve vegetations and with positive blood cultures led to diagnosis of a ruptured mycotic aneurysm. In three other cases CT with contrast enhancement demonstrated the presence of a left subdural haematoma and of two mass lesions which at neurosurgery were diagnosed as a cerebral abscess and a glioblastoma, both located in the right frontal lobe. In the ninth case, a 48 year old woman, occurrence of isolated sixth nerve paresis five weeks after her pure motor stroke prompted execution of MRI, which showed several hyperintense lesions on $T_{2}$

Table 2 Prevalence of cerebrovascular risk factors

\begin{tabular}{lll}
\hline & $\begin{array}{l}\text { Ischaemic lacunar } \\
\text { syndromes } \\
(n=88)\end{array}$ & $\begin{array}{l}\text { Non ischaemic } \\
\text { lacunar syndromes } \\
(n=9)\end{array}$ \\
\hline $\begin{array}{l}\text { Hypertension* } \\
\text { Previous TIA }\end{array}$ & $57(64 \cdot 8 \%)$ & $2(22 \cdot 2 \%)$ \\
$\begin{array}{l}\text { Hypertension and/or } \\
\text { previous TIA } \dagger\end{array}$ & $20(22 \cdot 7 \%)$ & 0 \\
$\begin{array}{l}\text { Diabetes } \\
\text { Hyperlipidaemia }\end{array}$ & $68(77 \cdot 3 \%)$ & $2(22 \cdot 2 \%)$ \\
Smoking & $25(19 \cdot 3 \%)$ & $1(11 \cdot 1 \%)$ \\
Cardiac disease & $30(34 \cdot 1 \%)$ & $2(22 \cdot 2 \%)$ \\
\hline
\end{tabular}

${ }^{*} \chi^{2}=4.55$ (with Yates correction); $p<0.05$.

$+\chi^{2}=9.73$ (with Yates correction); $p<0.005$ weighted images, with a pattern compatible with multiple sclerosis; this diagnosis was confirmed by the presence of oligoclonal bands in the CSF and by evidence of abnormal visual and somatosensory evoked responses.

Clinical findings are summarised in table 1 . None of our patients had impairment of consciousness, in keeping with the clinical diagnosis of lacunar syndrome. Although non-sudden onset of symptoms was more frequent among lacunar infarcts, this difference did not reach statistical significance. Only a small proportion of patients in both groups experienced headache, nor did the type of lacunar syndrome allow to separate non ischaemic from ischaemic cases. The prevalence of cerebrovascular risk factors is presented in table 2. Only hypertension was significantly $(p<0.05)$ more frequent among ischaemic patients than among those with lacunar syndromes due to other causes. Sensitivity of this risk factor in detecting ischaemic patients was $64.8 \%$ and specificity $77.8 \%$, with a positive predictive value of $96.6 \%$ and a negative predictive value of $18 \cdot 4 \%$.

\section{Discussion}

According to several studies, lacunar strokes account for $13-24 \cdot 6 \%$ of all cerebral infarcts. ${ }^{13-15}$ Although several isolated case reports have underscored that different aetiologies can give rise to lacunar syndromes (including intracerebral haemorrhage, ${ }^{16}$ subdural haematoma and multiple sclerosis, ${ }^{10}$ tumours ${ }^{17}$ and infections,${ }^{18}$ ) the prevalence of these cases has, to our knowledge, never been studied prospectively in a consecutive series of patients with very recent onset of their first-ever "lacunar stroke". Weisberg studied a series of patients referred for CT scan because of recent onset of rapidly developing hemiparesis or hemiplegia and found non ischaemic causes of symptoms in as many as seven out of 33 patients, three of whom had a haemorrhage. ${ }^{10}$ Also Rascol et al limited 
their study to pure motor hemiplegia $;{ }^{19}$ in their report, two out of 30 cases had a capsular haemorrhage and no patient had a non vascular aetiology. In a study of 186 patients with lacunar syndrome admitted at their institution, including 38 with pseudobulbar palsy, Loeb et al found non ischaemic causes in 18 cases, seven of whom were haemorrhagic. ${ }^{20}$ In their community-based study, Bamford et al found three cases of intracerebral haemorrhage among 104 patients with their first-ever lacunar stroke, 93 of whom had CT scan within four weeks; ${ }^{15}$ these authors did not mention the occurrence of non vascular aetiologies. Lacunar syndromes due to intracerebral haemorrhage may be less rare than expected, since Mori et $a l^{1}$ observed them in 19 out of 174 consecutive patients with spontaneous intracerebral haemorrhage, and Huang et al found that two out of 11 patients with lacunar syndrome and CT evidence of a brainstem lesion had a haemorrhage. ${ }^{22}$ The importance of identifying cases with cerebral haemorrhage is stressed by the fact that lacunar strokes are commonly benign, leaving only mild to moderate disability; consequently, these patients are often potential candidates for long-term prophylactic antithrombotic treatment. ${ }^{23}$ Moreover, gradual or fluctuating onset of the symptoms, which was observed in $38 \%$ of our patients and even in $62 \%$ of lacunar strokes in the Harvard Stroke Registry, ${ }^{14}$ may suggest a progressing stroke and lead to prompt heparin treatment. Clinical findings are of little value in the individuation of patients with lacunar infarction; the Allen diagnostic score, ${ }^{24}$ which allows the probability of stroke being due to infarction or haemorrhage to be calculated from a number of clinical variables, indicates a $>90 \%$ probability of cerebral infarction if it is lower than 4 . Yet, all our non ischaemic patients had scores $<4$, owing to the paucity of their symptoms, which were compatible with a typical lacunar syndrome. A history of hypertension was significantly more frequent among our ischaemic patients, and its positive predictive value in diagnosing lacunar infarction was $96.6 \%$. However, two of our hypertensive patients had intracerebral haemorrhage; Mori et $a l^{21}$ found hypertension in 17 of their 19 haemorrhagic patients presenting with lacunar syndrome, and six of the seven haemorrhagic patients with pure motor hemiparesis reported by Weisberg and Wall ${ }^{25}$ were also hypertensive. Moreover, the negative predictive value associated with the presence of hypertension was very low in our study group, with an inadequate diagnostic yield. Although none of our non ischaemic patients had experienced previous transient ischaemic attacks (TIA), the combination of this feature with hypertension would only marginally improve the positive predictive value of the diagnosis of lacunar infarct to $97.1 \%$, and the negative predictive value would remain very low $(25 \cdot 9 \%)$. On the contrary, prompt non enhanced CT scan allowed immediate diagnosis of intracerebral haemorrhage in five of our patients and demonstrated a mass lesion in three others, two of whom could receive effective neurosurgical treatment. Since acute onset of lacunar syndromes due to non ischaemic causes is not rare, early CT scanning is warranted in patients with "lacunar strokes", particularly if they are normotensive, and whenever antithrombotic treatment is considered.

\section{References}

1 Fisher CM. Lacunes: small deep cerebral infarcts. Neurology 1965;15:775-84.

2 Fisher CM. Capsular infarcts. The underlying vascular lesions. Arch Neurol 1979;36:65-73.

3 Fisher CM, Curry HB. Pure motor hemiplegia of vascular origin Arch Neurol 1965;13:30-44.

4 Fisher CM. Pure sensory stroke involving face, arm and leg. Neurology 1965;15:76-80.

5 Fisher CM, Cole M. Homolateral ataxia and crural paresis: a vascular syndrome. J Neurol Neurosurg Psychiatry 1965;28: 48-55.

6 Fisher CM. A lacunar stroke: The dysarthria-clumsy hand syndrome. Neurology 1967;17:614-7.

7 Fisher CM. Lacunar strokes and infarcts: a review. Neurology 1982;32:871-6.

8 Santamaria J, Graus F, Rubio F, Arbizu T, Peres J. Cerebral infarction of the basal ganglia due to embolism from the heart. Stroke 1983;14:911-4.

9 Aleksic SN, George AE. Pure motor hemiplegia with occlusion of the extracranial carotid artery. $J$ Neurol Sci 1973;19:331-9.

10 Weisberg LA. Computed tomography and pure motor hemiparesis. Neurology 1979;29:490-5.

11 Kobatake K, Shinohara Y. Ataxic hemiparesis in patients with primary pontine hemorrhage. Stroke 1983;14:762-4.

12 Tapia JF, Kase CS, Sawyer RH, Mohr JP. Hypertensive putaminal hemorrhage presenting as pure motor hemiparesis. Stroke 1983;14:505-6.

13 Gross CR, Kase CS, Mohr JP, Cunningham SC, Baker WE. Stroke in South Alabama: incidence and diagnostic features-a-a population based study. Stroke 1984;15:249-55.

14 Mohr JP, Caplan LR, Melski JW, et al. The Harvard cooperative stroke registry: a prospective registry. Neurology 1978;28 754-62.

15 Bamford J, Sandercock P, Jones L, Warlow C. The natural history of lacunar infarction: the Oxfordshire community stroke project. Stroke 1987;18:545-51.

16 Mori E, Yamadori A, Kudo Y, Tabuchi M. Ataxic hemiparesis from small capsular hemorrhage. Arch Neurol 1984;41:1050-3.

17 Fisher M, Recht L. Brain tumor presenting as pure motor stroke. Neurology 1988;38 (suppl 1):341.

18 Barinagarementeria $\mathrm{F}$, Del Brutto $\mathrm{OH}$, Otero E. Ataxic hemiparesis from cysticercosis. Arch Neurol 1988;45:246.

19 Rascol A, Clanet M, Manelfe C, Guiraud B, Bonafe A. Pure motor hemiplegia: CT study of 30 cases. Stroke 1982;13:11-17.

20 Loeb C, Gandolfo C, Mancardi GL, Primavera A, Tassinari T. The lacunar syndromes: a review with personal contribution. In: Lechner H, Meyer JS, Ott EO, eds. Cerebrovascular Disease: Research and Clinical Management, vol 1. Amsterdam: Elsevier Science Publishers, 1986.

21 Mori E, Tabuchi M, Yamadori A. Lacunar syndrome due to intracerebral hemorrhage. Stroke 1985;16:454-9.

22 Huang CY, Woo E, Yu YL, Chan FL. Lacunar syndromes due to brainstem infarct and hemorrhage. J Neurol Neurosurg Psychiatry 1988;51:509-15.

23 Antiplatelet Trialists' Collaboration. Secondary prevention of vascular disease by prolonged antiplatelet treatment. $\mathrm{Br}$ Med J 1988;296:320-31.

24 Allen CMC. Clinical diagnosis of the acute stroke syndrome. $Q J$ Med 1983;208:515-23.

25 Weisberg LA, Wall M. Small capsular hemorrhages. Clinicalcomputed tomographic correlations. Arch Neurol 1984; 41:1255-7. 